\title{
Originalien
}

Nervenarzt 2021 · 92:479-486

https://doi.org/10.1007/s00115-020-00975-0

Online publiziert: 10. August 2020

(c) Der/die Autor(en) 2020

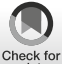

Eckhard Frick' · Philip Ziemer ${ }^{1}$ Stephan Heres ${ }^{2} \cdot$ Karl Ableidinger $^{3} \cdot$ Franz Pfitzer $^{4}$. Arndt Büssing ${ }^{5}$

' Klinik und Poliklinik für Psychosomatische Medizin und Psychotherapie, Klinikum rechts der Isar, Technische Universität München, München, Deutschland

${ }^{2}$ kbo-Isar-Amper-Klinikum gGmbH, Akademisches Lehrkrankenhaus der LMU München, Haar bei München, Deutschland

${ }^{3}$ Landesklinikum Amstetten-Mauer, Mauer, Österreich

${ }^{4}$ Klinik Chiemseewinkel, Seeon-Seebruck, Deutschland

${ }^{5}$ Professur für Lebensqualität, Spiritualität und Coping, Universität Witten/Herdecke, Witten, Deutschland

\section{Spirituelle Kompetenz in Psychiatrie und Psychotherapie - Hindernisse und Erfolgsfaktoren}

wichtiges Thema in der Psychotherapie halten, aber nur ca. ein Fünftel routinemäßig eine sp Anamnese erhebt. Eine solche sp Kurzanamnese ist insbesondere im US-amerikanischen Raum in vielen Bereichen etabliert [24] und wird zunehmend auch im deutschsprachigen Raum praktiziert [12]. Die eigene Nähe zur $R$ korreliert positiv mit der Tendenz, die Kurzanamnese in die Therapie einzubinden. Bei ca. einem Drittel der Therapeuten besitzt $R$ einen wichtigen Stellenwert innerhalb der eigenen Weltanschauung („personal bias“; [17]).

Freund und Kollegen [11] befragten psychiatrische Weiterbildungsermächtigte in Deutschland. Insgesamt bezeichneten sich $60 \%$ der antwortenden Weiterbildungsermächtigten selbst als $r$ und/oder sp. Der Großteil der Befragten misst der Integration von $s p$ Fragestellungen in die Facharztweiterbildung eine hohe Bedeutung bei. Eine Signalwirkung kommt der Richtlinie des österreichischen Gesundheitsministeriums zu [15], die eindringlich vor der Gefahr des Missbrauchs sp Methoden in der Psychotherapie warnt.

Ein aktueller Handbuchbeitrag [21] beklagt den psychiatrischen Nachholbedarf im Vergleich zu SpC innerhalb der Palliativmedizin; es existiere noch immer kein Konsens über psychiatrische $S p C$-Kernkompetenzen. Stattdessen wer- de versucht, $r / s p$ Patientenbelange an die Seelsorge zu delegieren.

Die aktuelle Studie erfragt erstmals im deutschen Sprachraum sowohl einzelne Bereiche der selbsteingeschätzten Spiritual-Care-Kompetenz $(S p C K)$ aller psychiatrisch-psychotherapeutischen Berufsgruppen als auch das globale Urteil dieser Experten bezüglich der $S p K$ ihrer Berufsgruppe. Mittels eines standardisierten und validierten Befragungsinstrumentes werden die folgenden Forschungsfragen bearbeitet:

1. Wie schätzen Psychiater und andere an der psychiatrischen Akutversorgung beteiligte Berufsgruppen ihre Zuständigkeit bzw. Nichtzuständigkeit für $S p C$ ein?

\begin{tabular}{|ll}
\hline \multicolumn{2}{|l}{ Abkürzungen } \\
\hline$K$ & Kompetenz \\
\hline$R$ & Religion/Religiosität \\
\hline$r$ & Religiös \\
\hline$S C C Q$ & $\begin{array}{l}\text { Spiritual Care Competency } \\
\text { Questionnaire }\end{array}$ \\
\hline$S p$ & Spiritualität \\
\hline$S p$ & Spirituell \\
\hline$S p C$ & Spiritual Care \\
\hline$S p C K$ & Spiritual-Care-Kompetenz \\
\hline$S p K$ & Spirituelle Kompetenz \\
\hline
\end{tabular}


Tab. 1 Soziodemographische und berufsbezogene Daten der psychiatrisch tätigen Personen

\begin{tabular}{|c|c|c|c|c|c|}
\hline & Alle & Ärzte & Pflegende & Andere & $\begin{array}{l}\text { Signifikanz } \\
\text { (ANOVA/ } X^{2} \text { ) }\end{array}$ \\
\hline Anzahl $^{a}$ & 647 & 75 & 391 & 119 & - \\
\hline Alter (Jahre) & $41,5 \pm 11,8$ & $45,9 \pm 10,5$ & $40,9 \pm 11,9$ & $40,8 \pm 11,6$ & 0,003 \\
\hline Frauen (\%) & 73 & 73 & 69 & 84 & 0,007 \\
\hline Berufstätigkeit (Jahre) & $19,0 \pm 12,4$ & $17,1 \pm 10,4$ & $20,3 \pm 12,9$ & $15,8 \pm 11,5$ & 0,001 \\
\hline $\begin{array}{l}\text { Arbeitszeit (Stunden/ } \\
\text { Woche) }\end{array}$ & $35,4 \pm 9,2$ & $39,7 \pm 10,5$ & $36,1 \pm 8,3$ & $30,4 \pm 9,2$ & $<0,0001$ \\
\hline $\begin{array}{l}\text { Berufliche Zufriedenheit } \\
(1-5)\end{array}$ & $4,0 \pm 0,8$ & $4,1 \pm 0,7$ & $3,9 \pm 0,8$ & $4,1 \pm 0,7$ & 0,023 \\
\hline \multicolumn{5}{|l|}{ Religionszugehörigkeit (\%) } & \multirow[t]{5}{*}{0,001} \\
\hline Katholisch & 53 & 40 & 54 & 56 & \\
\hline Evangelisch & 8 & 16 & 5 & 14 & \\
\hline Andere & 7 & 8 & 6 & 7 & \\
\hline Keine & 33 & 36 & 35 & 23 & \\
\hline Gläubig (\%) & 54 & 62 & 52 & 56 & n.s. \\
\hline $\begin{array}{l}\text { Häufigkeit Gebet/ } \\
\text { Meditation (1-4) }\end{array}$ & $2,3 \pm 1,1$ & $2,6 \pm 1,1$ & $2,2 \pm 1,1$ & $2,6 \pm 1,1$ & $<0,0001$ \\
\hline \multicolumn{6}{|l|}{ Kompetenzen (SCCQ) } \\
\hline Wahrnehmungs- $K$ & $1,9 \pm 0,6$ & $2,1 \pm 0,6$ & $1,9 \pm 0,6$ & $2,1 \pm 0,6$ & $<0,0001$ \\
\hline Team-Spirit & $0,8 \pm 0,6$ & $0,8 \pm 0,6$ & $0,8 \pm 0,6$ & $0,7 \pm 0,5$ & n.s. \\
\hline Dokumentations- $K$ & $0,6 \pm 0,7$ & $0,5 \pm 0,6$ & $0,7 \pm 0,7$ & $0,5 \pm 0,6$ & 0,002 \\
\hline $\begin{array}{l}\text { Selbsterfahrung und } \\
\text { Öffnung }\end{array}$ & $1,0 \pm 0,7$ & $1,1 \pm 0,7$ & $0,9 \pm 0,6$ & $1,2 \pm 0,7$ & $<0,0001$ \\
\hline $\begin{array}{l}\text { Wissen über andere } \\
\text { Religionen }\end{array}$ & $1,7 \pm 0,7$ & $1,6 \pm 0,6$ & $1,7 \pm 0,7$ & $1,7 \pm 0,6$ & n.s. \\
\hline Gesprächsführungs- $K$ & $2,3 \pm 0,7$ & $2,5 \pm 0,6$ & $2,2 \pm 0,7$ & $2,4 \pm 0,6$ & $<0,0001$ \\
\hline Empowerment- $K$ & $1,8 \pm 0,6$ & $1,9 \pm 0,7$ & $1,8 \pm 0,6$ & $1,9 \pm 0,7$ & 0,020 \\
\hline Umsetzungshindernisse & $1,2 \pm 0,8$ & $1,0 \pm 0,8$ & $1,3 \pm 0,7$ & $1,0 \pm 0,7$ & $<0,0001$ \\
\hline
\end{tabular}

2. Von welchen Variablen wird die Einschätzung fehlender Zuständigkeiten für $S p C$ beeinflusst?

3. Wie unterscheiden sich die Berufsgruppen hinsichtlich der Gründe, die der Umsetzung von $S p C$ im Wege stehen?

\section{Studiendesign und Untersu- chungsmethoden}

\section{Probanden und Rekrutierung}

\section{Rekrutierung}

Psychiatrisch-psychotherapeutisch Tätige im Isar-Amper-Klinikum München (Rücklaufquote ca. $31 \%$ ), im Landesklinikum Mauer (39\%) und in drei Kliniken des Trägers Gesundheitswelt Chiemgau $(60 \%)$ wurden persönlich kontaktiert. Interessierte erhielten den Fragebogen in Papierform und wurden in der
Folge einmal erinnert. Alle Teilnehmenden erklärten ihre Bereitschaft zur anonymisierten Verwendung der Daten.

\section{Soziodemographische und berufsbezogene Daten}

Alle Probanden wurden nach ihrem Geschlecht, Alter, Familienstand, Religionszugehörigkeit sowie nach Merkmalen ihrer beruflichen Tätigkeit gefragt. Außerdem konnten sie sich äußern, ob sie sich als aktiv gläubige Person bezeichnen würden (4-stufige Zustimmungsskala: ja, unbedingt; ja, etwas; eher nein; nein, gar nicht) oder wie häufig sie beten oder meditieren (regelmäßig; hin und wieder; eher selten; gar nicht).

\section{SpC-Kompetenzen}

Zur Beurteilung der selbsteingeschätzten SpCK wurde der Spiritual Care Compe- tence Questionnaire (SCCQ) verwendet [13]. Dieser nutzt 26 Items und differenziert 7 Dimensionen (Cronbachs a 0,73-0,86):

1. Wahrnehmungs- $K$ (z. B. Bedürfnisse der Angehörigen oder Patienten wahrnehmen),

2. Team-Spirit (z. B. Austausch im Team über das Thema oder eigene $S p$ ),

3. Dokumentations-K (z.B. Kenntnis von Instrumenten zur Erfassung $s p$ Bedürfnisse, Fähigkeit zur nachvollziehbaren Dokumentation),

4. Selbsterfahrung und proaktive Öffnung (z. B. Vertiefung eigener $S p$, Zuwendung zu Patienten, um deren $s p$ Bedürfnisse anzusprechen),

5. Wissen über andere Religionen (z. B. Kenntnisse zu Besonderheiten verschiedener Religionsgemeinschaften und deren Berücksichtigung),

6. Gesprächsführungs-K (z. B. offenes Gespräch über existenzielle oder religiöse Themen führen können),

7. proaktive Empowerment-K (z.B. Ermöglichung der Teilnahme an $r$ Feiern, geeigneter Rahmen für $s p$ Gespräche).

Umsetzungshindernisse für $S p C$ wurden mit 4 zusätzlichen Items erfasst (Cronbachs a 0,72: Ich weiß zu wenig über $R / S p$, um mich kompetent einbringen zu können; keine Zeit für $r / s p$ Themen; kein geeigneter Raum vorhanden, um geschützt über $r / s p$ Themen zu sprechen; empfinde mich für $r / s p$ Themen als nicht zuständig). Für die Zustimmung bzw. Ablehnung der entsprechenden Aussagen des SCCQ wurde eine 4-stufige Skalierung gewählt (0 - stimmt nicht; 1 - stimmt kaum;2-stimmt eher;3-stimmt genau).

\section{Statistische Analysen}

Die deskriptive, Varianz, Korrelations(Spearman $\rho$ ) und stufenweise Regressionsanalysen wurde mit dem Programm SPSS in der Version 23.0 durchgeführt. Es wurde ein Signifikanzniveau von $<0,01$ gewählt. Für die Korrelationsanalysen wurde eine Korrelationskoeffizient $r>0,5$ als starke Korrelation angesehen, $r$ zwischen 0,3 und 0,5 als moderate Korrelation, $r$ zwischen 0,2 und 0,3 als 
Nervenarzt 2021 · 92:479-486 https://doi.org/10.1007/s00115-020-00975-0

(c) Der/die Autor(en) 2020

\section{E. Frick $\cdot$ P. Ziemer $\cdot$ S. Heres $\cdot$ K. Ableidinger $\cdot$ F. Pfitzer $\cdot$ A. Büssing}

\section{Spirituelle Kompetenz in Psychiatrie und Psychotherapie - Hindernisse und Erfolgsfaktoren}

\section{Zusammenfassung}

Hintergrund. Ebenso wie die World Psychiatric

Association (WPA) und andere nationale psychiatrische Fachgesellschaften hat auch die Deutsche Gesellschaft für Psychiatrie und Psychotherapie, Psychosomatik und Nervenheilkunde (DGPPN) ein Positionspapier zu Religiosität und Spiritualität (R/Sp) in Psychiatrie und Psychotherapie veröffentlicht, in dem sie Patientenzentrierung und spirituelle Kompetenz (SpK) der psychiatrischen Berufe fordert. Es ist bekannt, dass Kompetenzmangel das wichtigste Hindernis für die Implementierung von Spiritual Care $(\mathrm{SpC})$ in die klinische Praxis darstellt.

Fragestellung. Ziel der vorliegenden Studie ist die praxisnahe Untersuchung der SpK in Psychiatrie und Psychotherapie. Wie schätzen psychiatrisch Tätige die SpK ihrer eigenen Berufsgruppe ein und welche Variablen beeinflussen dieses Urteil?

Material und Methoden. Insgesamt 391 psychiatrische Pflegekräfte, 75 Psychiater, 119 Therapeuten verschiedener Professionen und 62 andere $(n=647)$ aus Krankenhäusern in Deutschland und Österreich füllten den SpC Competency Questionnaire (SCCQ) aus. Ergebnisse. Pflegekräfte, ältere und spirituell erfahrenere Personen schätzen die SpK der eigenen Berufsgruppe vergleichsweise höher ein und meinen seltener, diesbezüglich nicht zuständig zu sein. Pflegende nennen häufiger als andere Berufsgruppen das Fehlen geeigneter Räumlichkeiten als Hindernis für die Implementierung von SpC. Höhere Ein- schätzung der SpK der eigenen Berufsgruppe geht mit höheren Werten in den SCCQFaktoren "Selbsterfahrung und proaktive Öffnung ", "Team-Spirit", „Wahrnehmungs-/Dokumentationskompetenz" einher.

Diskussion. Die Zuständigkeit der Gesundheitsberufe für $\mathrm{SpC}$ in Psychiatrie und Psychotherapie wird unter den deutschsprachigen psychiatrischen Berufsgruppen noch kontrovers diskutiert. Dies hängt mit mangelnder Kompetenz in diesem Feld zusammen.

Schlüsselwörter

Kompetenz - Spiritual Care · Psychiatrie . Psychotherapie $\cdot$ Implementierung

\section{Spiritual competence in psychiatry and psychotherapy—Barriers and success factors}

\section{Abstract}

Background. Just as the World Psychiatric Association (WPA) and other national psychiatric societies, the German Association for Psychiatry, Psychotherapy and Psychosomatics (DGPPN) has published a position statement about religiosity and spirituality in psychiatry and psychotherapy, in which it demands patient orientation and spiritual competency in psychiatric professions. Previous research has shown that lack of competency is the major barrier against implementing spiritual care into clinical practice.

Objective. The aim of this study was to examine spiritual care in psychiatry and psychotherapy. An evaluation of how health professionals in psychiatry gauge the spiritual care competency of their professional group and which variables influence this judgement. Material and methods. A total of 391 psychiatric nursing personnel, 75 psychiatrists, 119 therapists from diverse professions and 62 others, i.e. 647 working in German and Austrian hospitals completed the German version of the spiritual care competency questionnaire (SCCQ).

Results. Nursing personnel, older and spiritually more experienced persons gauged the spiritual competency of their own professional group comparatively higher and judged less frequently that they have no responsibility in this field. Nursing personnel reported the lack of suitable rooms as a barrier against implementation of spiritual care more often than other professional groups. Judging the spiritual competency of one's own professional group higher is associated with higher values in the SCCQ factors self-experience and proactive opening up, team spirit, perception and documentation competency.

Conclusion. The responsibility of healthcare professions for spiritual care in psychiatry and psychotherapy is still a controversial issue among German-speaking psychiatric professional groups. This is partially due to a lack of competency in this domain.

Keywords

Spiritual care - Competency · Psychiatry . Psychotherapy $\cdot$ Implementation schwache Korrelation und $r<0,2$ als keine oder vernachlässigbare Korrelation.

\section{Ergebnisse}

\section{Beschreibung der teilnehmenden Personengruppe}

Es konnten Datensätze von 647 Personen ausgewertet werden: $12 \%$ Ärzte, $60 \%$ Pflegende, $18 \%$ andere (Therapeuten, Psychologen). Diese Berufsgruppen unterscheiden sich signifikant sowohl in
Bezug auf Alter und Geschlecht, Arbeitszeit und Berufstätigkeit als auch bezüglich der Religionszugehörigkeit und der Häufigkeit $r$ Praxis (•Tab. 1). Im Schnitt haben 33\% keinerlei Religionszugehörigkeit, der Anteil der Katholiken ist bei den Ärzten deutlich geringer und der Anteil der Protestanten etwas höher; die Hälfte der untersuchten Personen bezeichnet sich als "gläubig“.

\section{Empfundene Zuständigkeit für SpC}

In der Gesamtgruppe stimmen 27,5\% nicht $\mathrm{zu}$, eine besondere $S p K \mathrm{zu}$ haben, $37,0 \%$ stimmen kaum zu, 29,0\% stimmen eher zu und 6,5\% stimmen genau zu. Der verneinenden Aussage, dass ihre Berufsgruppe nicht für $S p C$ zuständig sei, wurde in der Gesamtgruppe zumeist nicht zugestimmt: $37,0 \%$ stimmten nicht $\mathrm{zu}, 27,7 \%$ stimmen kaum zu, 22,3\% stimmen eher zu und 13,0\% stimmen genau zu. 
Tab. 2 Zugeschriebene SpK/Zuständigkeit in den Berufsgruppen (Zustimmungsscores 0-3)

Meine Berufsgruppe hat Meine Berufsgruppe ist besondere SpK nicht für $S p C$ zuständig

\begin{tabular}{l|l}
\hline Insgesamt & MW \\
& SD \\
& $n$
\end{tabular}

\section{Profession}

Arzt $\mathrm{MW}$

0,91

0,93

SD

SD

0,86

0,95

Pflege

MW

70

72

SD

$\mathrm{SD}$
$n$

Andere

MW

1,17

1,12

0,88

1,03

324

SD

1,12

1,00

1,08

$n$

0,94

103

F-Wert

$p$-Wert

2,46

0,087

1,33

Altersgruppen

MW

1,02

1,13

SD

0,87

1,02

n

141

120

31-40 Jahre

MW

1,07

1,32

SD

0,94

1,05

$n$

140

131

41-50 Jahre

MW

1,23

1,03

SD

0,84

1,07

$n$

130

118

$>50$ Jahre

MW

1,27

0,89

SD

0,91

0,98

n

168

152

F-Wert

2,76

4,39

$p$-Wert

0,041

0,005

Gebet/Meditation

\begin{tabular}{|c|c|c|c|}
\hline \multirow[t]{3}{*}{ Nicht/selten } & MW & 1,08 & 1,27 \\
\hline & SD & 0,89 & 1,03 \\
\hline & $n$ & 317 & 281 \\
\hline \multirow{3}{*}{$\begin{array}{l}\text { Hin und wieder/ } \\
\text { regelmäßig }\end{array}$} & $\mathrm{MW}$ & 1,23 & 0,92 \\
\hline & SD & 0,90 & 1,03 \\
\hline & $n$ & 282 & 254 \\
\hline \multicolumn{2}{|l|}{ F-Wert } & 4,32 & 15,40 \\
\hline \multicolumn{2}{|l|}{$p$-Wert } & 0,038 & $<0,0001$ \\
\hline
\end{tabular}

Es zeigte sich ein Trend für besonders geringe Zustimmung bei den Ärzten, dass sie eine besondere $S p K$ hätten (• Tab. 2).

Für beide Aussagen gab es altersassoziierte Unterschiede: Mit zunehmendem Alter wurde eine besondere SpK gese- hen bzw. wurde die Nichtzuständigkeit verneint (• Tab. 2). Geschlechtsassoziierte Unterschiede ließen sich nicht finden (nicht dargestellt).

Personen, die häufiger beten oder meditieren, zeigen eine etwas höhere $\mathrm{Zu}$ - stimmung, eine besondere $S p K$ zu haben als Personen, die dies nicht oder nur selten tun würden. Die Nichtzuständigkeit wird signifikant eher von den nicht bzw. wenig betenden oder meditierenden Personen benannt als von den "religiöseren“ Personen (• Tab. 2).

\section{SpC-Kompetenzen der Berufsgruppen}

Die selbsteingeschätzten $S p C K$ zeigen deutliche Ausprägungsunterschiede (- Tab. 1). Die Gesprächsführungs-K wird als hoch eingeschätzt (besonders von Ärzten, signifikant geringer von Pflegenden), ebenso die Wahrnehmungs- $K$ (signifikant geringer bei Pflegenden) und Empowerment- $K$ (mit geringen Unterschieden zwischen den Berufsgruppen). Deutlich geringe $K$-Zuschreibungen finden sich für Selbsterfahrung und Öffnung (signifikant höher bei den anderen Berufsgruppen und gering bei den Pflegenden), Team-Spirit (ohne signifikante Unterschiede zwischen den Berufsgruppen) und besonders gering für Dokumentations- $K$ (besonders gering bei Ärzten und anderen Berufsgruppen).

Signifikante geschlechtsassoziierte Unterschiede lassen sich nicht finden (nicht dargestellt). Mit zunehmendem Alter steigen $K$ im Bereich Selbsterfahrung und Öffnung $(\mathrm{F}=13,5 ; p<0,0001)$, Empowerment- $K \quad(\mathrm{~F}=6,1 ; p<0,0001)$ und das Wissen über andere Religionen $(\mathrm{F}=4,0 ; p=0,008)$ an.

Die Einschätzung als gläubiger bzw. wenig/nichtgläubiger Mensch ist mit unterschiedlich hoch ausgeprägten $S p C K$ assoziiert: Selbsterfahrung und Öffnung $(\mathrm{F}=109,9 ; \quad p<0,0001)$ und Wahrnehmungs- $K(\mathrm{~F}=13,3 ; p<0,0001)$ sind bei Gläubigen deutlich stärker ausgeprägt, etwas weniger ausgeprägt auch Empowerment- $K(\mathrm{~F}=4,6 ; p=0,033)$, Gesprächsführungs- $K \quad(\mathrm{~F}=4,5 ; \quad p=0,034) \quad$ und Dokumentations $K \quad(\mathrm{~F}=4,2 ; p=0,041)$, während die Umsetzungshindernisse ( $\mathrm{F}=10,3 ; p=0,001)$ bei den Wenig-/ Nichtgläubigen signifikant größer waren. Lediglich für Team-Spirit und Wissen über andere Religionen zeigen sich keine Gruppenunterschiede. 
Tab. 3 Prädiktoren derempfundenen besonderen SpKbzw. derNichtzuständigkeit (schrittweise Regressionsanalysen)

\begin{tabular}{|c|c|c|c|c|c|}
\hline \multirow{2}{*}{\multicolumn{3}{|c|}{$\begin{array}{l}\mathbf{R}^{2} \\
\text { Abhängige Variable: Item } 57 \text { (besondere } S p K)^{\mathrm{a}} \\
\mathrm{F}=37,3 ; p<0,0001\end{array}$}} & Beta & \multirow[t]{2}{*}{$\mathbf{T}$} & \multirow[t]{2}{*}{$p$} \\
\hline & & & & & \\
\hline \multirow[t]{5}{*}{ Modell 4} & (Konstante) & - & - & 1,319 & 0,188 \\
\hline & $\begin{array}{l}\text { Selbsterfahrung und } \\
\text { Öffnung }\end{array}$ & 0,13 & 0,234 & 5,399 & $<0,0001$ \\
\hline & Team-Spirit & 0,18 & 0,169 & 4,090 & $<0,0001$ \\
\hline & Dokumentations- $K$ & 0,20 & 0,121 & 2,916 & 0,004 \\
\hline & Wahrnehmungs- $K$ & 0,21 & 0,118 & 2,605 & 0,009 \\
\hline \multicolumn{6}{|c|}{$\begin{array}{l}\text { Abhängige Variable: Item } 59 \text { (Nichtzuständigkeit) }^{b} \\
\mathrm{~F}=98,0 ; p<0,0001\end{array}$} \\
\hline \multirow[t]{3}{*}{ Modell 2} & (Konstante) & - & - & 6,721 & $<0,0001$ \\
\hline & Umsetzungshindernisse & 0,24 & 0,375 & 8,675 & $<0,0001$ \\
\hline & $\begin{array}{l}\text { Selbsterfahrung und } \\
\text { Öffnung }\end{array}$ & 0,28 & $-0,235$ & $-5,443$ & $<0,0001$ \\
\hline \multicolumn{6}{|c|}{$\begin{array}{l}\text { Dargestellt ist die hierarchische Bedeutsamkeit der eingeschlossenen Einflussvariablen auf die Aus- } \\
\text { prägung der abhängigen Variablen „besondere SpK" bzw. "Nichtzuständigkeit” } \\
\text { aichtsignifikant im Modell: Alterskohorte, Häufigkeit Gebet/Meditation, Empowerment- } K \text {, Umset- } \\
\text { zungshindernisse } \\
{ }^{b} \text { Nichtsignifikant im Modell: Alterskohorte, Häufigkeit Gebet/Meditation, Empowerment- } K \text {, Wahr- } \\
\text { nehmungs- } K \text {, Team-Spirit, Dokumentations- } K\end{array}$} \\
\hline
\end{tabular}

\section{Empfundene Zuständigkeit für $S p C$ und Einflussvariablen}

Wie hängt die empfundene Zuständigkeit bzw. Nichtzuständigkeit mit den $S p C K$ und berufsbezogenen Variablen zusammen? In Korrelationsanalysen ergibt sich kein signifikanter Zusammenhang mit Berufsjahren, Arbeitszeit oder beruflicher Zufriedenheit $(r<0,12)$. Die selbsteingeschätzte "besondere $S p K^{\text {“ }}$ der Berufsgruppe ist moderat assoziiert mit Wahrnehmungs- $K$, TeamSpirit und Selbsterfahrung und Öffnung $(r>0,30, p<0,0001)$; die Nichtzuständigkeit hat einen moderaten Zusammenhang mit Umsetzungshindernissen $(r=0,49 ; p<0,0001)$ sowie moderat invers mit Selbsterfahrung und Öffnung $(r=-0,412, p<0,0001)$.

Die Bedeutsamkeit dieser assoziierten Variablen wird mithilfe einer Regressionsanalyse ausgewertet (बTab. 3). Für die besondere $\mathrm{SpK}$ sind $4 S p C K$ als Prädiktoren bedeutsam (21\% Varianzerklärung), wobei Selbsterfahrung/Öffnung alleine bereits $13 \%$ der Varianz erklärt. Für die Nichtzuständigkeit sind Umsetzungshindernisse und Selbsterfahrung/ Öffnung signifikante Prädiktoren, die $28 \%$ der Varianz erklären (Umsetzungshindernisse bereits $24 \%$ ). In keinem der

\section{Diskussion}

Die in der Einleitung formulierten Forschungsfragen können wir folgendermaßen beantworten:

1. Eine besondere Zuständigkeit für $S p C$ wird eher nicht gesehen (etwas weniger von Ärzten), aber eben auch nicht eine besondere Nichtzuständigkeit. Hier drückt sich eine deutliche Zurückhaltung und Unsicherheit zur eigenen Positionierung aus. Eine „professionelle Neutralität“ ist auch bei US-amerikanischen Allgemeinmedizinern belegbar [7].

2. Für die Einschätzung einer besonderen $S p K$ der eigenen Berufsgruppe sind Selbsterfahrung und proaktive Öffnung der beste Prädiktor, während die Nichtzuständigkeit besonders mit Umsetzungshemmnisses begründet wird, also eine eher abwehrende Haltung den $r / s p$ Themen gegenüber. Hier spielt also die eigene (ablehnende) Grundhaltung eine bedeutende Rolle.

3. Für die Einschätzung einer besonderen $S p K$ der eigenen Berufsgruppe ist Selbsterfahrung/proaktive Öffnung der beste Prädiktor, während die Nichtzuständigkeit besonders mit Umsetzungshemmnissen begründet wird, also eine eher abwehrende Haltung den $r / s p$ Themen gegenüber widerspiegelt. Hier spielt also die eigene (ablehnende) Grundhaltung eine bedeutende Rolle. Die einzelnen $S p C K$ sind unterschiedlich stark ausgeprägt und zeigen signifikante Unterschiede zwischen den in der Psychiatrie beschäftigen Berufsgruppen. Innerhalb der SpCK sind insbesondere Selbsterfahrung/ Öffnung und Wahrnehmungs- $K$ bei Gläubigen deutlich stärker ausgeprägt, während Hemmnisse eher bei den Wenig-/Nichtgläubigen zu finden waren. Hinsichtlich der Gründe, die der Umsetzung von $S p C$ im Wege stehen, unterscheiden sich die Berufsgruppen kaum. Die Pflegenden geben signifikant häufiger an, es sei "kein geeigneter Raum vorhanden", die Ärzte, sie könnten „nicht alles lösen". 


\begin{tabular}{|c|c|c|c|c|c|c|c|c|c|}
\hline \multicolumn{2}{|c|}{ Scores 0-3 } & $\begin{array}{l}\text { Unange- } \\
\text { nehm, über } \\
s p \text { Themen } \\
\text { zu reden }\end{array}$ & $\begin{array}{l}\text { Wechsle Thema, } \\
\text { wenn Patient } \\
\text { auf } r \text { Themen zu } \\
\text { sprechen kommt }\end{array}$ & $\begin{array}{l}\text { Habe kei- } \\
\text { ne Zeit, r/sp } \\
\text { Themen an- } \\
\text { zusprechen }\end{array}$ & $\begin{array}{l}\text { Hätte gerne mehr Zeit, } \\
\text { um mit Patienten über } \\
\text { deren sp Bedürfnisse } \\
\text { zu sprechen }\end{array}$ & $\begin{array}{l}\text { Kein ge- } \\
\text { eigneter } \\
\text { Raum vor- } \\
\text { handen }\end{array}$ & $\begin{array}{l}\text { Weiß } \\
\text { zu } \\
\text { wenig }\end{array}$ & $\begin{array}{l}\text { Nicht } \\
\text { zu- } \\
\text { stän- } \\
\text { dig }\end{array}$ & $\begin{array}{l}\text { Kann } \\
\text { nicht } \\
\text { alles } \\
\text { lösen }\end{array}$ \\
\hline \multirow{2}{*}{$\begin{array}{l}\text { Insgesamt } \\
(n=584)\end{array}$} & MW & 0,65 & 0,01 & 1,17 & 1,31 & 1,16 & 1,20 & 1,21 & 0,22 \\
\hline & SD & 0,85 & 0,13 & 1,05 & 0,96 & 1,10 & 0,96 & 1,03 & 0,74 \\
\hline \multirow{2}{*}{$\begin{array}{l}\text { Ärzte } \\
(n=75)\end{array}$} & MW & 0,53 & 0,00 & 1,16 & 1,38 & 0,88 & 1,01 & 0,96 & 0,69 \\
\hline & SD & 0,89 & 0,00 & 1,09 & 1,02 & 0,94 & 0,94 & 0,94 & 1,15 \\
\hline \multirow{2}{*}{$\begin{array}{l}\text { Pflegende } \\
(n=381)\end{array}$} & MW & 0,68 & 0,01 & 1,21 & 1,29 & 1,29 & 1,27 & 1,32 & 0,06 \\
\hline & SD & 0,86 & 0,14 & 1,04 & 0,97 & 1,12 & 0,95 & 1,04 & 0,40 \\
\hline \multirow{2}{*}{$\begin{array}{l}\text { Andere } \\
(n=118)\end{array}$} & MW & 0,62 & 0,02 & 1,04 & 1,35 & 0,90 & 1,10 & 1,00 & 0,45 \\
\hline & SD & 0,80 & 0,13 & 1,04 & 0,89 & 1,04 & 1,00 & 1,03 & 1,04 \\
\hline \multicolumn{2}{|l|}{ F-Wert } & 1,04 & 0,39 & 1,16 & 0,40 & 8,66 & 2,92 & 6,68 & 33,79 \\
\hline \multicolumn{2}{|l|}{$p$-Wert } & n.s. & n.s. & n.s. & n.s. & $<0,0001$ & 0,055 & 0,001 & $<0,0001$ \\
\hline
\end{tabular}

Die meisten Autoren stimmen darin überein, dass $S p C$ die Seelsorge nicht ersetzen, dass aber die dementsprechende Sensibilisierung und Kompetenzvertiefung der Gesundheitsberufe die Zusammenarbeit mit der Seelsorge erleichtern kann [22, 23]. Im Unterschied zum sehr restriktiven österreichischen Positionspapier [15] sieht das DGPPNPositionspapier [29] einen größeren Spielraum vor, um die positiven, resilienzfördernden Möglichkeiten der Einbeziehung von $R / S p$ zu gewährleisten, ohne zu versäumen, auf mögliche Gefahren hinzuweisen. Ebenso wie das DGPPN-Positionspapier [29] versteht die Mehrheit der Literatur $S p$ als den im Vergleich zu $R$ umfassenderen Begriff. Eine pflegewissenschaftliche Konzeptanalyse [5] subsummiert $S p$ als das wertebasierte Denken der Patienten, ihre Fähigkeit zur Interaktion mit anderen, ihre Suche nach Lebenssinn und Verbundenheit („connectedness“) mit anderen Menschen und mit dem transzendenten Heiligen. Psychiatrisches $S p C$ könne tröstliche Effekte haben und das Coping der Patienten fördern. Eine aktuelle psychotherapeutische Studie [32] belegt die Zusammenhänge zwischen $R / S p$ einerseits und der Suche nach dem Lebenssinn und fordert, dass Psychotherapeuten ihren Patienten die Exploration von $R / S p$ ermöglichen, gleichzeitig jedoch ihre professionellen Grenzen wahren sollten.

SpCKim Sinne der in der vorliegenden Studie gemessenen Selbsteinschätzungen umfasst Wahrnehmung-, Gesprächsführungs- und Dokumentations- $K$ [13], klinisch gesprochen: die Fähigkeit zur Erhebung einer proaktiven sp Anamnese [29], die im deutschen Sprachraum noch zurückhaltend praktiziert wird [17]. Hingegen erklärten $93 \%$ der von Curlin et al. [8] befragten US-amerikanischen Psychiater (vs. $53 \%$ in anderen Facharztgruppen), es sei angemessen, $R / S p$ aktiv in der Anamnese zu explorieren. Eine polnische Studie [4] begründet die Zurückhaltung gegenüber der proaktiven sp Anamnese mit dem ethischen Argument des Patientenschutzes vor Grenzverletzungen und mit dem Mangel an diesbezüglichem Training.

Der häufig diskutierte $r / s p$,gap“ zwischen psychiatrischen Patienten und ihren Behandlern $[6,19]$ oder auch der weltanschaulichen „Kultur“ von Kliniken [11] wird dadurch vertieft, dass deutschsprachige Fachpersonen ihre $S p K$ oft überschätzen und einschlägige Weiter- und Fortbildungsangebote noch immer dünn gesät sind [14]. Weder eine undifferenzierte Pathologisierung des $r / s p$ Patientenerlebens noch die naive Idealisierung jedweder $S p$ sind in therapeutischer Hinsicht hilfreich [9]. Vielmehr setzt sich die Haltung durch, dass $S p K$ zum kultursensiblen Umgehen mit Diversität gehört, das von den psychiatrischen Berufen zu fordern ist [16, 26]. Psychiatrisch Tätige brauchen SpK und psychopathologisches Urteilsvermögen, um $r / s p$ Bedürfnisse einerseits, religiöse
Wahnbildungen u. a. Krankheitssymptome andererseits $\mathrm{zu}$ unterscheiden und differenziert beantworten $\mathrm{zu}$ können [9]. Wenn z.B. das Verstummen $r / s p$ Äußerungen zum Erfolgskriterium für die antipsychotische Medikation wird, verschließt sich ein unter Coping-Gesichtspunkten wichtiger Zugang zum Patienten [10]. Dazu bedarf es einer Verbesserung der Ausbildung [27, 28], der Weiterbildung $[11,28]$ und der Fortbildung [20, 25, 28, 31].

\section{Limitationen}

Das Thema eines „personal Bias“ spielte in unserer Studie eine ebenso große Rolle wie in früheren Untersuchungen. Mit einer Selbstselektion ist schon bei der Entscheidung zur (Nicht-)Teilnahme zu rechnen. Dieser Selektionseffekt geht allerdings auch mit einem Sensibilisierungseffekt einher: Das Bewusstwerden der eigenen $r / s p$ Orientierung fördert das Einnehmen einer professionellen Haltung zu den $r / s p$ Patientenbedürfnissen [10]. Es erleichtert dem Umgang mit sich wandelnden Definitionen von $R / S p$ : Während früher jeglicheSpim Kontext von $R$ gesehen oder gar beide Terme gleichgesetzt wurden, wird nun zunehmend akzeptiert, dass es $s p$ Bedürfnisse und Orientierungen auch außerhalb institutionalisierter $R$ geben kann. Die damit gegebene begriffliche Unschärfe ist Vor- und Nachteil zugleich: Frühere Modelle wie die Gemeinschaftsspiritualität 
$r$ gebundener Krankenschwestern oder die Gleichsetzung von wissenschaftlicher und atheistischer Haltung weichen weniger globalen und damit stärker personenbezogenen Zugangsweisen.

\section{Fazit für die Praxis}

- SpK wird im Sinne der Patientenzentrierung gefordert (Matching zwischen sp Bedürfnissen psychisch Kranker und der diesbezüglichen Sensibilisierung der Behandler).

- Mithilfe des SCCQ können Maßnahmen der Aus-, Fort- und Weiterbildung personen- und gruppenbezogen konzipiert und evaluiert werden.

- Verbesserung der SpK in der Psychiatrie kann durch Überarbeitung und Ergänzung von Lerninhalten und -zielen sowie durch Organisationsund Kulturentwicklung psychiatrischpsychotherapeutischer Institutionen erreicht werden.

- Zentral ist die Förderung spezifischer diagnostischer Fähigkeiten, um auf $r / s p$ Bedürfnisse und Krankheitssymptome differenziert einzugehen.

- Solange ein $r / s p$ „personal bias“ des Behandlers unbewusst bleibt, kann es die Wahrnehmung verzerren. Wird es thematisiert und professionell gehandhabt, fördert es die Patientenorientierung.

\section{Korrespondenzadresse}

Prof. Dr. med. Eckhard Frick, SJ, MA

Klinik und Poliklinik für Psychosomatische Medizin und Psychotherapie, Klinikum rechts der Isar, Technische Universität München Ismaninger Str. 22, 81675 München, Deutschland eckhard.frick@tum.de

Funding. Open Access funding provided by Projekt DEAL.

\section{Einhaltung ethischer Richtlinien}

Interessenkonflikt. E. Frick, P. Ziemer, S. Heres, K. Ableidinger, F. Pfitzer und A. Büssing geben an, dass kein Interessenkonflikt besteht.

Für diesen Beitrag wurden von den Autoren keine Studien an Menschen oder Tieren durchgeführt. Für die aufgeführten Studien gelten die jeweils dort angegebenen ethischen Richtlinien. Von allen an der
Befragung Beteiligten liegt eine schriftliche Einverständniserklärung vor. Die Studie wurde von der Ethikkommission der TU München genehmigt (171/18S).

Open Access. Dieser Artikel wird unter der Creative Commons Namensnennung 4.0 International Lizenz veröffentlicht, welche die Nutzung, Vervielfältigung, Bearbeitung, Verbreitung und Wiedergabe in jeglichem Medium und Format erlaubt, sofern Sie den/die ursprünglichen Autor(en) und die Quelle ordnungsgemäß nennen, einen Link zur Creative Commons Lizenz beifügen und angeben, ob Änderungen vorgenommen wurden.

Die in diesem Artikel enthaltenen Bilder und sonstiges Drittmaterial unterliegen ebenfalls der genannten Creative Commons Lizenz, sofern sich aus der Abbildungslegende nichts anderes ergibt. Sofern das betreffende Material nicht unter der genannten Creative Commons Lizenz steht und die betreffende Handlung nicht nach gesetzlichen Vorschriften erlaubt ist, ist für die oben aufgeführten Weiterverwendungen des Materials die Einwilligung des jeweiligen Rechteinhabers einzuholen

Weitere Details zur Lizenz entnehmen Sie bitte der Lizenzinformation auf http://creativecommons.org/ licenses/by/4.0/deed.de.

\section{Literatur}

1. Abdulla A, Hossain M, Barla C (2019) Toward comprehensive medicine: listening to spiritual and religious needs of patients. Gerontol Geriatr Med 5:2333721419843703

2. Appleby A, Swinton J, Wilson P (2019) Spiritual care training and the GP curriculum: Where to now? Educ Prim Care 30:194-197

3. Balboni MJ, Sullivan A, Amobi A et al (2013) Why is spiritual care infrequent at the end of life? Spiritual care perceptions among patients, nurses, and physicians and the role of training. J Clin Oncol 31:461-467

4. Charzyńska E, Heszen-Celińska I (2020) Spirituality and mental health care in a religiously homogeneous country: definitions, opinions, and practices among Polish mental health professionals. J Relig Health 59:113-134

5. Clark M, Emerson A (2020) Spirituality in psychiatric nursing: a concept analysis. J Am Psychiatr Nurses Assoc. https://doi.org/10.1177/ 1078390320902834

6. Cook CCH, Powell A, Sims A et al (2011) Spiritualityand secularity: professional boundaries in psychiatry. Ment Health Relig Cult 14:35-42

7. Curlin FA, Chin MH, Sellergren SA et al (2006) The association of physicians' religious characteristics with their attitudes and self-reported behaviors regarding religion and spirituality in the clinical encounter. Med Care 44:446-453

8. Curlin FA, Lawrence RE, Odell $S$ et al (2007) Religion, spirituality, and medicine: psychiatrists' and other physicians' differing observations, interpretations, and clinical approaches. Am J Psychiatry 164:1825-1831

9. Demling JH (2017) "Gesunde" und leidvolle Religiosität. Versuch einer psychiatrisch-psycho(patho)logischen Abgrenzung. Spirit Care 7:81-87

10. Elliott R, Wattis J, Chirema K et al (2020) Mental health nurses' understandings and experiences of providing care for the spiritual needs of service users: a qualitative study. J Psychiatr Ment Health Nurs 27:162-171

11. Freund H, Böhringer S, Utsch M et al (2018) Religiositätund Spiritualitätin derFacharztweiterbildung Eine Umfrage bei den Weiterbildungsermächtigten für Psychiatrie und Psychotherapie. Nervenarzt 89:539-545

12. Frick E (2019) Spirituelle Anamnese. In: Roser T (Hrsg) Handbuch der Krankenhausseelsorge. Vandenhoeck \& Ruprecht, Göttingen, S291-300

13. Frick E, Theiss M, Rodrigues Recchia D et al (2019) Validierung einer deutschsprachigen Skala zur Messung der Spiritual Care Kompetenz. Spirit Care 8:193-207

14. Hofmann L, Walach $H$ (2011) Spirituality and religiosity in psychotherapy - a representative survey among German psychotherapists. Psychother Res 21:179-192

15. Jansky-Denk G, Sagl M, Weiss S (2014) Richtlinie für Psychotherapeutinnen und Psychotherapeuten zur Frage der Abgrenzung der Psychotherapie von esoterischen, spirituellen und religiösen Methoden des Bundesministeriums für Gesundheit auf Grundlage eines Gutachtens des Psychotherapiebeirates vom 17. Juni 2014. In: Österreichisches Bundesministerium für Arbeit $\mathrm{S}$, Gesundheit und Konsumentenschutz (BMASGK) (ed), Wien

16. Jimenez RR, Thal W (2020) Culturally competent mental health care. Nurs Made Incred Easy J 18:46-49

17. Marquardt M, Demling JH (2016) Psychotherapie und Religion: Eine repräsentative Umfrage unter Psychotherapeuten in Süddeutschland. Psychother Psychosom Med Psychol 66:473-480

18. Moreira-Almeida A, Sharma A, Rensburg BJV et al (2015) WPA Position statement on spirituality and religion in psychiatry. http:// www.wpanet.org/uploads/Position_Statement/ WPA\%20position\%20Spirituality\%20statement $\% 20$ final\%20version_rev2\%20on\%20Spirituality. pdf.Zugegriffen: 8. Mai 2016

19. Van Nieuw Amerongen-Meeuse JC, SchaapJonker H, Schuhmann C et al (2018) The "religiosity gap" in a clinical setting: experiences of mental health care consumers and professionals. Ment Health Relig Cult 21:737-752

20. Pearce MJ, Pargament KI, Oxhandler HK et al (2019) A novel training program for mental health providers in religious and spiritual competencies. Spiritual Clin Pract 6:73-82

21. Peteet J (2020) Spirituality/religion and end-of-life care. In: Rosmarin DH, Koenig HG (Hrsg) Handbook of spirituality, religion, and mental health. Elsevier, London, S201-217

22. Plante TG (2020) Consultation with religious professionals and institutions. In: Falender CA, Shafranske EP (Hrsg) Consultation in psychology: a competency-based approach. American Psychological Association, Washington, DC, S 221-237

23. Poncin E, Niquille B, Jobin G et al (2020) What motivates healthcare professionals' referrals to chaplains, and how to help them formulate referrals that accurately reflect patients' spiritual needs? J Health Care Chaplain 26:1-15

24. Puchalski C (2006) Spiritual assessment in clinical practice. PSYCH 36:150-155

25. Rasinski KA, Kalad YG, Yoon JD et al (2011) An assessment of US physicians' training in religion, spirituality, and medicine. Med Teach 33:944-945

26. Richards PS, Bergin AE (2014) Toward religious and spiritual competency for mental health professionals. In: Richards PS, Bergin AE (Hrsg) Handbook of psychotherapy and religious diversity, 2. Aufl. 
American Psychological Association, Washington, DC, S3-19

27. Smothers ZPW, Tu JY, Grochowski C et al (2019) Efficacy of an educational intervention on students' attitudes regarding spirituality in healthcare: a cohort study in the USA. BMJ Open 9:e26358-e26358

28. Taverna M, Sattel H, Berberat Pet al (2019) A survey on the integration of spiritual care in medical schools from the German speaking faculties. Adv MedEduc Pract 10:1009-1019

29. Utsch M, Anderssen-Reuster U, Frick E et al (2017) Empfehlungen zum Umgang mit Religiosität und Spiritualität in Psychiatrie und Psychotherapie. Spirit Care 6:141-146

30. Vieten C, Scammell S (2015) Spiritual and religious competencies in clinical practice: guidelines for psychotherapists and mental health professionals. New Harbinger Publications, Oakland, CA

31. Woodhouse R, Hogan KF (2020) 'out on the edge of my comfort': trainee counsellor/psychotherapists' experiences of spirituality in therapy - a qualitative exploration. Couns Psychother Res 20:173-181

32. YoonE, Cabirou L, HoepfAetal (2020) Interrelations of religiousness/spirituality, meaning in life, and mental health. Couns Psychol Q. https://doi.org/ 10.1080/09515070.2020.1712651

\section{Leitthemenübersicht Der Nervenarzt}

Der Nervenarzt bietet Ihnen umfassende und aktuelle Beiträge zu interessanten Themenschwerpunkten aus allen Bereichen der Neurologie, Psychiatrie und Nervenheilkunde.

\section{Überblick Leitthemenhefte 2019}

- 1/19 Originalienheft

- 2/19 Genetik in Neurologie und Psychiatrie

- 3/19 Psychisch kranke Eltern und ihre Kinder

- 4/19 Originalienheft

- 5/19 Originalienheft

- 6/19 Neuroonkologie

- 7/19 Stationssetting in der Psychiatrie

- 8/19 Personalisierte Therapien in der Neurologie

- 9/19 Quo vadis Alzheimer's disease?

- 10/19 Update Schlaganfall

- 11/19 Forschung für eine personenzentrierte Psychiatrie und Psychotherapie

- 12/19 Patientenmonitoring 4.0

\section{Überblick Leitthemenhefte 2020}

- 1/20 Schizophrenie

- 2/20 Originalienheft

- 3/20 Bipolare Störungen

- 4/20 Genspezifische Therapien neurologischer Erkrankungen

- 5/20 Palliativmedizin bei psychiatrischen Erkrankungen

- 6/20 Versorgungsforschung

- 7/20 Update ADHS

- 8/20 Placebo

- 9/20 Stigmatisierung psychischer Erkrankungen

- 10/20 Schlaganfall Versorgungs-Realität

- 11/20 Psychiatrie und Psychotherapie in der sozialen Lebenswelt

- 12/20 Darm und Gehirn

Alle Inhalte der Zeitschrift finden Sie unter www.springermedizin.de/der-nerven-

arzt.

SpringerMedizin.de bietet Ihnen Zugang zu allen elektronisch verfügbaren Ausgaben Ihrer Zeitschrift - unabhängig davon, seit wann Sie die Zeitschrift abonniert haben.

Möchten Sie ein bereits erschienenes Heft nachbestellen? Einzelne Ausgaben können
Sie direkt bei unserem Kundenservice zum Preis von je EUR 44,- zzgl. Versandkosten beziehen:

Springer Nature Customer Service Center Kundenservice Zeitschriften

Tiergartenstr. 15, 69121 Heidelberg

Tel.: +49 $6221345-4303$

Fax: +496221 345-4229

E-Mail: leserservice@springernature.com

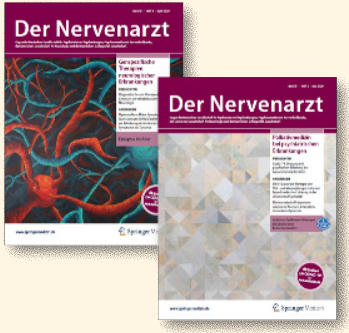

